\section{Effectiveness and safety of tocilizumab for the treatment of refractory systemic sclerosis associated interstitial lung disease: a case series}

We read with great interest the results of the double-blind phase 2 faSScinate clinical trial, ${ }^{1}$ in which there was encouraging (although not statistically significant) numerical improvement in skin thickening and evidence of less decline in lung function in patients with systemic sclerosis (SSc) treated with tocilizumab (TCZ) compared with those receiving placebo. Initial investigations with TCZ in patients with SSc demonstrated improvements in skin sclerosis and polyarthritis. ${ }^{23}$

Scleroderma-associated interstitial lung disease (SSc-ILD) is a severely debilitating complication with high mortality in extensive disease. There is no approved disease-modifying treatment, and few effective treatment options are available. One of the most urgent needs is to determine which drugs can be useful as a rescue treatment in patients who do not respond to conventional immunosuppressants (cyclophosphamide (CYC) or mycophenolate mofetil (MMF)). In this sense, TCZ appears as one of the most promising candidates and an ongoing 2-year randomised phase 3 trial of TCZ-SC $162 \mathrm{mg}$ in SSc is under way (ClinicalTrials.gov identifier NCT02453256).

Based on this preliminary evidence, ${ }^{1-3}$ we have evaluated TCZ (off-label use) as a rescue therapy in nine selected patients with progressive SSc-ILD (evidence of clinical and functional decline) despite previous treatment with low-medium prednisone doses, immunosuppressants and rituximab (RTX). They were treated with a compassionate use of TCZ for at least 6 months. In all cases, written informed consent was obtained from the patients, and the off-label use of TCZ (and previously of RTX) was approved by our local health authorities. ${ }^{4}$

The median durations of SSc and ILD were 8 years (range: 2-15 years) and 7 years (range: 2-12 years), respectively. All cases corresponded to fibrosing non-specific interstitial pneumonia. Progressive interstitial lung disease (ILD) was defined when there was a worsening of $\geq 10 \%$ in per cent predicted forced vital capacity (\%pFVC) or $\geq 15 \%$ in per cent predicted diffusing capacity for carbon monoxide corrected for haemoglobin (\%pDLCO) during the follow-up (over 1 year).

Previous or ongoing therapies for SSc-ILD included MMF (100\%), CYC (67\%), azathioprine (11\%) and RTX (100\%). In all cases, the time elapsed since the last dose of CYC was greater than 2 years and 6 months in the case of RTX. The mean number of RTX cycles previously administered was $3 \pm 1.7$ (range: $1-6$ ): in four patients (44\%) RTX was discontinued due to adverse events (mainly respiratory or urinary infections and/or transient neutropenia) and in the remaining (56\%) due to inefficacy.

TCZ was administered intravenously in two patients (at a dose of $8 \mathrm{mg} / \mathrm{kg}$ monthly) and subcutaneously in the remaining 7 (162 mg weekly). In all cases, it was administered with MMF (eight patients received $2 \mathrm{~g}$ /day and one patient received $1 \mathrm{~g} /$ day). Seven $(78 \%)$ patients received concomitant treatment with prednisone ( $\leq 5 \mathrm{mg} /$ day). Ongoing therapy with MMF and oral prednisone remained initially unchanged in all cases.

The baseline clinical features and outcome of these patients are summarised in table 1 . At the end of the follow-up period (median 12 months; IQR 25th-75th: 6-33 months), only four patients (44\%) were still in treatment. In the other five patients $(56 \%)$ TCZ was discontinued, due to serious adverse events in one case and due to inefficacy in the other four cases. One of these,

Table 1 Baseline clinical features and outcome of our nine patients with refractory systemic sclerosis-associated interstitial lung disease treated with tocilizumab

\begin{tabular}{|c|c|c|c|c|c|c|c|c|c|}
\hline Patient number & 1 & 2 & 3 & 4 & 5 & 6 & 7 & 8 & 9 \\
\hline Age (years)/sex & $59 / \mathrm{F}$ & $40 / \mathrm{F}$ & $64 / \mathrm{F}$ & $63 / \mathrm{F}$ & $57 / \mathrm{F}$ & $53 / \mathrm{F}$ & $52 / \mathrm{F}$ & $60 / \mathrm{F}$ & $62 / F$ \\
\hline SSc duration (years) & 15 & 9 & 12 & 6 & 4 & 6 & 2 & 6 & 10 \\
\hline SSc cutaneous subset & Diffuse & Limited & Diffuse & Limited & Diffuse & Limited & Limited & Diffuse & Diffuse \\
\hline Chest HRCT pattern of ILD & Fibrosing NSIP & Fibrosing NSIP & Fibrosing NSIP & Fibrosing NSIP & Fibrosing NSIP & Fibrosing NSIP & Fibrosing NSIP & Fibrosing NSIP & Fibrosing NSIP \\
\hline ILD duration (years) & 12 & 4 & 12 & 6 & 4 & 6 & 2 & 6 & 9 \\
\hline Autoantibodies & $\begin{array}{l}\text { Scl-70 (+) } \\
\text { Ro52 (-) }\end{array}$ & $\begin{array}{l}\text { ACA (+) } \\
\text { Ro52: ND }\end{array}$ & $\begin{array}{l}\text { Scl-70 (+) } \\
\text { Ro52: ND }\end{array}$ & $\begin{array}{l}\text { ACA (+) } \\
\operatorname{Ro52}(-)\end{array}$ & $\begin{array}{l}\text { Scl-70 (+) } \\
\text { Ro52 (-) }\end{array}$ & $\begin{array}{l}\text { ACA (+) } \\
\text { Ro52: ND }\end{array}$ & $\begin{array}{l}\text { Scl-70 (+) } \\
\text { Ro52 (-) }\end{array}$ & $\begin{array}{l}\text { Scl-70 (+) } \\
\text { Ro52 (+) }\end{array}$ & $\begin{array}{l}\text { Scl-70 (+) } \\
\text { Ro52 (-) }\end{array}$ \\
\hline $\begin{array}{l}\text { Previous or ongoing } \\
\text { therapies for SSC-ILD }\end{array}$ & $\begin{array}{l}\text { CYC IV, MMF, RTX } \\
\text { and PDN. }\end{array}$ & MMF and RTX. & $\begin{array}{l}\text { CYC IV, MMF, RTX } \\
\text { and PDN. }\end{array}$ & $\begin{array}{l}\text { AZA, CYC IV, MMF, } \\
\text { RTXand PDN. }\end{array}$ & MMF and RTX. & $\begin{array}{l}\text { CYC IV, MMF, RTX } \\
\text { and PDN. }\end{array}$ & MMF, RTX and PDN. & $\begin{array}{l}\text { CYC IV, MMF, RTX } \\
\text { and PDN. }\end{array}$ & $\begin{array}{l}\text { CYC IV, MMF, RTX } \\
\text { and PDN. }\end{array}$ \\
\hline No of RTX cycles & 4 & 1 & 4 & 3 & 2 & 5 & 1 & 1 & 6 \\
\hline $\begin{array}{l}\text { Follow-up after first dose } \\
\text { of TCZ (months) }\end{array}$ & 23 & 8 & 34 & 33 & 7 & 6 & 8 & 12 & 18 \\
\hline $\begin{array}{l}\text { Lung responses to TCZ } \\
\text { therapy* }\end{array}$ & $\begin{array}{l}\text { Pre-TCZ/Post-TCZ } \\
\text { \%pCVF: } 61.7 / 56.3 \\
\text { (STB). } \\
\text { \%pTLC: } 89.1 / 62.6 . \\
\text { \%pDLCO: } 30.5 / 34.6 \\
\text { (STB). } \\
\text { 6MWT: } 420 \mathrm{~m} / 393 \mathrm{~m} . \\
\text { HRCT: STB. }\end{array}$ & $\begin{array}{l}\text { Pre-TCZ/Post-TCZ } \\
\text { \%pCVF: } 77.3 / 80.4 \\
\text { (STB). } \\
\text { \%pTLC: } 88.3 / 89.1 . \\
\text { \%pDLCO: } 41.4 / 48.1 \\
\text { (IMPR). } \\
\text { 6MWT: } 330 \mathrm{~m} \\
\text { 1360 m. } \\
\text { HRCT: STB. }\end{array}$ & $\begin{array}{l}\text { Pre-TCZ/Post-TCZ } \\
\text { \%pCVF: } 78.8 / 73.7 \\
\text { (STB). } \\
\text { \%pTLC: } 75.2 / 79.8 \\
\text { \%pDLCO: } 47.1 / 50 \\
\text { (STB). } \\
\text { 6MWT: } 390 \mathrm{~m} / 399 \mathrm{~m} . \\
\text { HRCT: STB. }\end{array}$ & $\begin{array}{l}\text { Pre-TCZ/Post-TCZ } \\
\text { \%pCVF: } 103 / 101 \\
\text { (STB). } \\
\text { \%pTLC: } 93 / 88 . \\
\text { \%pDLCO: } 27 / 35 \\
\text { (IMPR). } \\
\text { 6MWT: } 388 \mathrm{~m} / 396 \mathrm{~m} . \\
\text { HRCT: STB. }\end{array}$ & $\begin{array}{l}\text { Pre-TCZ/Post-TCZ } \\
\text { \%pCVF: } 70.2 / 60.1 \\
\text { (W). } \\
\text { \%pTLC: } 80.7 / 66 . \\
\text { \%pDLCO: } 42.1 / 35.5 \\
\text { (W). } \\
\text { 6MWT: } 388 \mathrm{~m} / 396 \mathrm{~m} . \\
\text { HRCT:W. }\end{array}$ & $\begin{array}{l}\text { Pre-TCZ/Post-TCZ } \\
\text { \%pCVF: } 108 / 112 \\
\text { (STB). } \\
\text { \%pTLC: ND/ND. } \\
\text { \%pDLCO: } 69 / 52 \text { (W). } \\
\text { 6MWT: } 418 \mathrm{~m} / 335 \mathrm{~m} . \\
\text { HRCT: } W .\end{array}$ & $\begin{array}{l}\text { Pre-TCZ/Post-TCZ } \\
\text { \%pCVF: } 70 / 56 \text { (W). } \\
\text { \%pTLC: } 83 / 66 . \\
\text { \%pDLCO: } 61 / 31 \text { (W). } \\
\text { 6MWT: ND. } \\
\text { HRCT:W. }\end{array}$ & $\begin{array}{l}\text { Pre-TCZ/Post-TCZ } \\
\text { \%pCVF: } 59 / 49 \text { (W). } \\
\text { \%pTLC: } 63 / 57 . \\
\text { \%pDLCO: } 35 / 30 \text { (W). } \\
\text { 6MWT: ND. } \\
\text { HRCT:W. }\end{array}$ & $\begin{array}{l}\text { Pre-TCZ/Post-TCZ } \\
\text { \%pCVF: } 80 / 91 \text { (IMPR). } \\
\text { \%pTLC: } 91 / 92 \text {. } \\
\text { \%pDLCO: } 65 / 70 \text { (STB). } \\
\text { 6MWT: ND. } \\
\text { HRCT: ND. }\end{array}$ \\
\hline Adverse events & $\begin{array}{l}\text { Yes } \\
\text { herpeszoster: 1; } \\
\text { bacterial infections: } \\
\text { 3, needing } \\
\text { hospitalisation } \\
\text { in one of them } \\
\text { (osteomyelitis). }\end{array}$ & No & No & No & No & No & No & No & No \\
\hline $\begin{array}{l}\text { Discontinuation of the } \\
\text { treatment at the endpoint } \\
\text { of patient follow-up and } \\
\text { reason }\end{array}$ & $\begin{array}{l}\text { Yes, due to adverse } \\
\text { events. } \\
\text { In waiting list for } \\
\text { lung transplantation. }\end{array}$ & No & No & No & $\begin{array}{l}\text { Yes, due to inefficacy. } \\
\text { Autologous stem cell } \\
\text { transplantation }\end{array}$ & Yes, due to inefficacy. & Yes, due to inefficacy. & $\begin{array}{l}\text { Yes; died due to } \\
\text { progression of the ILD }\end{array}$ & No \\
\hline
\end{tabular}


four patients died due to progression of ILD. The frequency of adverse events was low, occurring in only one patient (11\%) who developed repeat infections including an osteomyelitis complicating a digital ulcer requiring hospitalisation.

Although it is difficult to draw any firm conclusions from these data, according with our experience in this small cohort, TCZ appears to be safe. Its effectiveness as a rescue treatment in patients with refractory SSc-ILD seems modest but not negligible, achieving an improvement or stabilisation of pulmonary function ${ }^{5}$ in $44 \%$ of patients.

Javier Narváez, ${ }^{1}$ Judit LLuch, ${ }^{1}$ Juan José Alegre Sancho, ${ }^{2}$ Maria Molina-Molina, ${ }^{3}$ Joan Miquel Nolla, ${ }^{1}$ Ivan Castellvi ${ }^{4}$

${ }^{1}$ Departments of Rheumatology, Hospital Universitario de Bellvitge, Barcelona, Spain ${ }^{2}$ Department of Rheumatology, Hospital Universitario Dr. Peset, Valencia, Spain ${ }^{3}$ Department of Pneumology, Hospital Universitario de Bellvitge, Barcelona, Spain

${ }^{4}$ Rheumatology Unit, Hospital de la Santa Creu i Sant Pau, Barcelona, Spain

Correspondence to Javier Narváez, Department of Rheumatology, Hospital Universitario de BellvitgeFeixa Llarga, Barcelona 08907, Spain; fjnarvaez@bellvitgehospital.cat

Handling editor Josef S Smolen

Contributors All authors had access to the data and meets the Uniform Requirements for Manuscripts Submitted to Biomedical journals criteria for authorship.

Funding The authors have not declared a specific grant for this research from any funding agency in the public, commercial or not-for-profit sectors.

Competing interests None declared.

Patient consent Not required.

Ethics approval The present report has been approved by of our institutional ethics committee (Clinical Research Ethics Committee of Bellvitge University Hospital-IDIBELL). Written informed consent was obtained from the patients. Their clinical records and information were anonymised prior to analysis. This study was conducted in accordance with the principles of the Declaration of Helsinki and the International Conference for Harmonization.

Provenance and peer review Not commissioned; internally peer reviewed.
Data sharing statement The authors confirm that all data underlying the findings are fully available without restriction. All relevant data are within the paper.

(c) Author(s) (or their employer(s)) 2019. No commercial re-use. See rights and permissions. Published by BMJ.

\section{D) Check for updates}

To cite Narváez J, LLuch J, Alegre Sancho JJ, et al. Ann Rheum Dis 2019;78:e123.

Received 16 September 2018

Accepted 20 September 2018

Published Online First 23 October 2018

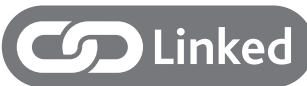

- https://doi.org/10.1136/annrheumdis-2018-214477

Ann Rheum Dis 2019;78:e123. doi:10.1136/annrheumdis-2018-214449

\section{REFERENCES}

1 Khanna D, Denton CP, Lin CJF, et al. Safety and efficacy of subcutaneous tocilizumab in systemic sclerosis: results from the open-label period of a phase II randomised controlled trial (faSScinate). Ann Rheum Dis 2018;77:212-20.

2 Shima Y, Kuwahara Y, Murota H, et al. The skin of patients with systemic sclerosis softened during the treatment with anti-IL-6 receptor antibody tocilizumab. Rheumatology 2010;49:2408-12.

3 Elhai M, Meunier M, Matucci-Cerinic M, et al. Outcomes of patients with systemic sclerosis-associated polyarthritis and myopathy treated with tocilizumab or abatacept: a EUSTAR observational study. Ann Rheum Dis 2013;72:1217-20.

4 Departament de Salut Generalitat de Catalunya, 2018. Dictamen de la Comissió Farmacoterapèutica de la Medicació Hospital ria deDispensació Ambulatòria sobre l'ús de rituximab, tocilizumab i abatacept per altractament fora d'indicació de l'esclerosi sistèmica refract ria al tractamenthabitual. http://catsalut.gencat.cat/web/.content/ minisite/catsalut/proveidors_professionals/medicaments_farmacia/phf_mhda/ informes_camse/esclerosi_sistemica/Dictamen-CAMS_ES_-web.pdf

5 Raghu G, Rochwerg B, Zhang Y, et al. An official ATS/ERS/JRS/ALAT clinical practice guideline: treatment of idiopathic pulmonary fibrosis. an update of the 2011 clinical practice guideline. Am J Respir Crit Care Med 2015;192:e3-e19.

6 Khanna D, Jahreis A, Lin CJF. Tocilizumab in systemic sclerosis-associated interstitial lung disease. Ann Rheum Dis 2019;78:e124. 